\title{
Author Correction: Development of a model- inference system for estimating epidemiological characteristics of SARS-CoV-2 variants of concern
}

Wan Yang (iD \& Jeffrey Shaman (D)

Correction to: Nature Communications https://doi.org/10.1038/s41467-021-25913-9, published online 22 September 2021.

The original version of this article contained errors in the Supplementary Information, equations S3 and S5.

Equation S3, line 4, was missing a term. The original read:

$$
\left\{\begin{array}{l}
\frac{d S}{d t}=\frac{R}{L}-\frac{b_{t} e_{t} m_{t} \beta_{t} I S}{N}-\varepsilon-v_{1}(t)-v_{2}(t) \\
\frac{d E}{d t}=\frac{b_{t} e_{t} m_{t} \beta_{t} I S}{N}-\frac{E}{Z}+\varepsilon \\
\frac{d I}{d t}=\frac{E}{Z}-\frac{I}{D} \\
\frac{d R}{d t}=\frac{I}{D}+v_{1}(t)+v_{2}(t)
\end{array}\right.
$$

This has been corrected to:

$$
\left\{\begin{array}{l}
\frac{d S}{d t}=\frac{R}{L}-\frac{b_{t} e_{t} m_{t} \beta_{t} I S}{N}-\varepsilon-v_{1}(t)-v_{2}(t) \\
\frac{d E}{d t}=\frac{b_{t} e_{t} m_{t} \beta_{t} I S}{N}-\frac{E}{Z}+\varepsilon \\
\frac{d I}{d t}=\frac{E}{Z}-\frac{I}{D} \\
\frac{d R}{d t}=\frac{I}{D}-\frac{R}{L}+v_{1}(t)+v_{2}(t)
\end{array}\right.
$$

Equation S5, line 2 was incorrectly written; the original read:

$$
\left\{\begin{array}{l}
\frac{d S_{i}^{A}}{d t}=\frac{R_{i}^{A}}{L_{i}^{A}}-\sum_{j} b_{t} e_{t} m_{t} c_{i j} \sum_{a} \frac{\beta_{j}^{A a} S_{I}^{A} I_{j}^{a}}{N^{a}}-\varepsilon_{i}-v_{i, 1}^{A}(t)-v_{i, 2}^{A}(t) \\
\frac{d E_{i}^{A}}{d t}=\sum_{j} b_{t} e_{t} m_{t} \sum_{a} \frac{\beta_{j}^{A a} j_{j}^{A} I_{j}^{a}}{N^{a}}-\frac{E_{i}^{A}}{Z_{i}^{A}}+\varepsilon_{i} \\
\frac{d I_{i}^{A}}{d t}=\frac{E_{i}^{A}}{Z_{i}^{A}}-\frac{I_{i}^{A}}{D_{i}^{A}} \\
\frac{d R_{i}^{A}}{d t}=\frac{I_{i}^{A}}{D_{i}^{A}}-\frac{R_{i}^{A}}{L_{i}^{A}}+v_{i, 1}^{A}(t)+v_{i, 2}^{A}(t)
\end{array}\right.
$$

This has been corrected to:

$$
\left\{\begin{array}{l}
\frac{d S_{i}^{A}}{d t}=\frac{R_{i}^{A}}{L_{i}^{A}}-\sum_{j} b_{t} e_{t} m_{t} c_{i j} \sum_{a} \frac{\beta_{j}^{A a} S_{j}^{A} I_{j}^{a}}{N^{a}}-\varepsilon_{i}-v_{i, 1}^{A}(t)-v_{i, 2}^{A}(t) \\
\frac{d E_{i}^{A}}{d t}=b_{t} e_{t} m_{t} \sum_{a} \frac{\beta_{i}^{A a} S_{i}^{A} I_{i}^{a}}{N^{a}}-\frac{E_{i}^{A}}{Z_{i}^{A}}+\varepsilon_{i} \\
\frac{d I_{i}^{A}}{d t}=\frac{E_{i}^{A}}{Z_{i}^{A}}-\frac{I_{i}^{A}}{D_{i}^{A}} \\
\frac{d R_{i}^{A}}{d t}=\frac{I_{i}^{A}}{D_{i}^{A}}-\frac{R_{i}^{A}}{L_{i}^{A}}+v_{i, 1}^{A}(t)+v_{i, 2}^{A}(t)
\end{array}\right.
$$

These corrections have been made to the Supplementary Information pdf. 
Published online: 16 December 2021

\section{Additional information}

Supplementary information The online version contains supplementary material available at https://doi.org/10.1038/s41467-021-27703-9.

(c) Open Access This article is licensed under a Creative Commons Attribution 4.0 International License, which permits use, sharing, adaptation, distribution and reproduction in any medium or format, as long as you give appropriate credit to the original author(s) and the source, provide a link to the Creative Commons license, and indicate if changes were made. The images or other third party material in this article are included in the article's Creative Commons license, unless indicated otherwise in a credit line to the material. If material is not included in the article's Creative Commons license and your intended use is not permitted by statutory regulation or exceeds the permitted use, you will need to obtain permission directly from the copyright holder. To view a copy of this license, visit http://creativecommons.org/licenses/by/4.0/.

(c) The Author(s) 2021 